Results CMPs and ICLCs showing typical features of either very immature or developing were found in the pulmonary vein sections of ten canine pulmonary veins model of persistent Atrial Fibrillation but not in the control group and were mainly identified in sections with a thick muscular sleeve. A positive immunostaining of CMPs was also demonstrated, the staining characteristic is similar to the $\mathrm{P}$ cell of the sinoatrial node, these cells maybe have the function of pacemaker.

Conclusion The pulmonary veins could host cardiac stem cell niches (CSCNs). Continuous rapid pacemaker can induce the differentiation of CMPs and ICLCs. CMPs could underlie the pacemaking activity of isolated pulmonary veins. ICLCs might act as supporting nurse cells of the cardiac niches and may be responsible for activation, commitment and migration of the stem cells out of the niches.

\section{[gw22-e0282] CARDIOMYOCYTE PROGENITORS IN CANINE PULMONARY VEINS MODEL OF PERSISTENT ATRIAL FIBRILLATION}

He Xian-zhi, Wang Haiyu, Shen Yan, Zhong Qinhua, Xue Jianfang Department Of Cardiology, Longgang District Central Hospital Of Shenzhen, Guangdong, China

10.1136/heartjnl-2011-300867.167

Background Recent studies suggested that various cell lineages exist within the pulmonary veins. However, the source and differentiation of such progenitor cells that may unmask a previously unknown source or mechanism of arrhythmogenesis has not been demonstrated. We hypothesised that this area could host cardiac stem cell niches (CSCNs)and continuous rapid pacemaker induced the differentiation of cardiomyocyte progenitors (CMPs) and interstitial Cajal-like cells (ICLCs)and could underlie the pacemaking activity of isolated pulmonary veins.

Objective This study aimed to discover the pathogenesis of focal atrial fibrillation (AF) originated from pulmonary veins, through observing the histological structure and special cells in canine pulmonary veins model of persistent Atrial Fibrillation.

Methods The pulmonary veins and the sinus node were obtained from 20 mongrel dogs (10AF and 10 control group) using light microscopy and transmission electron microscopy and the methods of immunohistochemistry on a transversal section of each pulmonary vein and Sinoatrial Node. Morphological and distribution analyses were performed manually and automatically. 